Macedonian Pharmaceutical Bulletin, 66 (Suppl 1) 143 - 144 (2020)

Online ISSN 1857 - 8969

UDC: $615.243: 615.451 .2 .014 .4$

DOI: 10.33320/maced.pharm.bull.2020.66.03.071

Short communication

\title{
Compounded omeprazole suspension - stable or not?
}

\author{
Ognjenka Rahić*, Edina Vranić, Jasmina Hadžiabdić, Merima Sirbubalo, Amina Tucak
}

Faculty of Pharmacy, University of Sarajevo, Zmaja od Bosne 8, 71000 Sarajevo, Bosnia and Herzegovina

\section{Introduction}

Omeprazole is a proton pump inhibitor commonly used in pediatric patients (Wensel, 2009). Pediatric patients are usually unable to swallow solid dosage forms and they need dose adjustment. Therefore, the dosage form of choice for this population is compounded liquid preparation.

Since pharmacies don't usually dispose of pure active substances, compounded liquid preparations are most commonly prepared from commercially available solid dosage forms, in a way that tablets are simply pulverized or capsule contents emptied, adding water or one of the commercially available vehicles (Haywood and Glass, 2013).

Considering the risks associated with the preparation and use of compounded preparations, the Chapter <795> of the US Pharmacopoeia states that the beyond-use date is 14 days for non-preserved aqueous oral formulations, if stored in the refrigerator. Preserved aqueous preparations can be stored for 35 days at controlled room temperature or in the refrigerator (USP, 2015).

\section{Materials and methods}

A review of the reference articles on the stability testing of compounded omeprazole suspensions has been conducted. Only articles with stability studies using stability indicating methods have been considered.

\section{Results and discussion}

Omeprazole suspension was prepared from pellets emptied from capsules with the addition of different excipients. Formulation 1 (F1) contained glycerol, carmellose sodium, simple syrup, and a combination of methyl and propyl paraben. The vehicle in the specified formulation was purified water. F1 was highly unstable (Milic et al., 2017). This is not a surprise, since the formulation does not contain agents to increase the $\mathrm{pH}$ value, which is necessary, given that the degradation of omeprazole occurs at the $\mathrm{pH}$ below 7.8. Given the stated, it is imperative to develop a formulation whose $\mathrm{pH}$ is above 8 .

Formulation $2(\mathrm{~F} 2)$ contained $8 \% \mathrm{w} / \mathrm{v}$ sodium bicarbonate, methyl and propyl paraben and propylene glycol. Vehicle was purified water. Sodium bicarbonate is included in F2. It maintains high $\mathrm{pH}$ while acting as a suspension agent (Whaley et al., 2012).

Formulation 3 (F3) was like F2, with the addition of xanthan gum. Xanthan gum, as a viscosity increasing agent, should increase the physical stability of the suspension. However, the modification of the rheological characteristics in this case did not lead to an improvement in the stability. F2 and F3 have proved to be stable 30 days in the refrigerator (Milic et al., 2017).

Formulation $4(\mathrm{~F} 4)$ of omeprazole suspension was prepared by dispersing omeprazole pellets in $8.4 \% \mathrm{w} / \mathrm{v}$ sodium bicarbonate solution. This formulation has been shown to be stable for up to 14

\footnotetext{
* ognjenka.rahic@ffsa.unsa.ba
} 
days at room temperature and up to 30 days in the refrigerator. In F4 the optimal $\mathrm{pH}$ is provided, due to the presence of sodium bicarbonate. Although no preservatives were added, microbial count was within the limits (Quercia et al., 1997).

Formulation 5 (F5) of omeprazole suspension is also made in $8.4 \% \mathrm{w} / \mathrm{v}$ sodium bicarbonate solution, but by dispersing pure omeprazole. The shelf life for this suspension was 45 days, if stored in the refrigerator (Allen, 2018). The fact that F5 was prepared with omeprazole pure substance, presumably resulted in a longer shelf life.

Formulation 6 (F6) of omeprazole suspension was prepared from pure omeprazole, which was subsequently mixed with SyrSpend SF Alka powder, and dispersed in purified water. F6 is stable for 92 days, if stored in the refrigerator. SyrSpend Alka powder consists of modified starch, calcium carbonate and sucralose. Modified starch acts as a suspending agent, calcium carbonate provides a $\mathrm{pH}$ above 8 . In addition, due to the unpleasant, bitter taste of omeprazole, which sodium bicarbonate in previous formulations further intensifies, the formulation of SyrSpend Alka included sweetener, which masks the unpleasant taste of the active substance (Whaley et al., 2012).

Formulation 7 (F7) of omeprazole suspension was obtained by reconstitution of omeprazole FIRST Compounding Kit. This kit consists of pure omeprazole and vehicle containing: strawberry aroma, benzyl alcohol, color, sweetener, poloxamer, propylene glycol, water, simethicone emulsion, sodium bicarbonate, sodium citrate, sucralose and xanthan gum. Beyond-use date for this suspension is 30 days, if kept in the refrigerator (Allen, 2019). Although, F7 contained more suspending agents, preservatives and buffers, it was not more stable than F6.

\section{Conclusion}

Stable omeprazole suspension is obtained by selecting the appropriate preparation process as well as the appropriate formulation. Suspensions prepared from pure omeprazole proved to be more stable than the ones prepared from omeprazole pellets.
The most stable suspension was F6 with the proven beyond-use date of 90 days in the refrigerator. The vehicle used consists of only three substances, which is a significant advantage over the others, because there is less possibility of interactions. It provides a $\mathrm{pH}$ above 8 , which is an absolute imperative from the aspect of the stability omeprazole. In addition, it contains sweetener, which improves the compliance of young patients. Additional advantage of this vehicle is that it does not contain sorbitol, ethanol, as well as propylene glycol and other preservatives. That makes it very suitable for newborns. When added simplicity of handling and quick preparation of this suspension in pharmacies, F6 can be considered as a formulation of choice for pediatric patients.

\section{References}

Allen, L.V., 2018. Omeprazole $1 \mathrm{mg} / \mathrm{mL}$ or $2 \mathrm{mg} / \mathrm{mL}$ oral suspension. US Pharm. 43, 47-48.

Allen, L.V., 2019. Omeprazole $2 \mathrm{mg} / \mathrm{mL}$ suspension. US Pharm. 44, Epub.

Haywood, A., Glass, B.D., 2013. Liquid dosage forms extemporaneously prepared from commercially available products - considering new evidence on stability. J. Pharm. Pharm. Sci. 16, 441-455.

Milic, J., Radojkovic, B., Jancic-Stojanovic, B., Draskovic, J., Mirasevic, S., Calija, B., 2017. Investigation of omeprazole stability in oral suspensions for pediatric use prepared extemporaneously from omeprazole capsules. Arh. farm. 67, 14-25.

Quercia, R.A., Fan, C., Liu, X., Chow, M.S.S., 1997. Stability of omeprazole in extemporaneously prepared oral liquid. Am. J. Healt-Syst. Pharm. 54, $1833-1836$

USP, 2015. The United States Pharmacopoeia 38th edition, The National Formulary 33rd edition, Rockville, Council of Experts and its Expert Committees: The United States Pharmacopoeial Convention.

Wensel, T.M., 2009. Administration of proton pump inhibitors in patients requiring enteral nutrition. Pharm. Therapeutics. 34, 143-160.

Whaley, P.A., Voudrie II, M.A., Sorenson, B., 2012. Stability of omeprazole in SyrSpend SF Alka (reconstituted). Int. J. Pharm. Compd. 16, 164-166. 